\section{Shedding Light and Electrons on the Lamellipodium: Imaging the Motor of Crawling Cells}

\author{
Emmanuel Vignal and Guenter P. Resch \\ Austrian Academy of Sciences, Institute of \\ Molecular Biology, Salzburg, Austria
}

\section{INTRODUCTION}

In a living organism, many cell types undergo extensive migration relative to their surrounding tissue to fulfill their biological function. Examples of this include fibroblast locomotion during wound healing, macrophage migration in response to a pathogen, and the extension of growth cones during neuronal organization, as well as in some important pathogenic states, for example, in the migration of metastatic cells (1).

The crawling movement of metazoan cells results from coordinated changes in cell shape, orchestrated by a continuous remodeling of the actin cytoskeleton $(2,3)$. Typically, migrating cells display a characteristic polarization with one or more broad, flat, ruffling lamellipodia extending in the direction of migration (the leading edge) and a retracting tail at the rear of the cell. Establishment of this cell shape, characteristic for a motile phenotype, requires a dynamic reorganization of the actin cytoskeleton, leading to the generation of forces necessary for cell body translocation (4) (Movie 1; Table 1).

Extension of the leading edge is the first and most important step of cell motility. Abercrombie and colleagues $(5,6)$ described the lamellipodium as the primary organelle of cell motility using time-lapse cinematography and electron microscopy. The lamellipodium is the motor where new actin filaments are generated. This was demonstrated in experiments performed by microinjecting fluorescent actin probes into fibroblasts and observing their dynamics during motility. Such experiments showed clearly that lamellipodia were the major "filament factory" of the cell (Movie 1; Table 1). The discovery of numerous regulatory proteins together with actin in the lamellipodium indicates that this organelle should not be considered solely as a polymerization machine but as a complex structure that is able to integrate signals (7).

\section{MICROSCOPY OF THE LAMELLIPODIUM}

Depending on cell type, lamellipodial morphology can vary from a single large, persistent lamellipodium in the case of keratocytes to several unstable lamellipodia, as for fibroblasts, epithelial cells, leukocytes, and neuronal processes. A lamellipodium can vary in breadth from 1 to $5 \mu \mathrm{m}$ and can exhibit highly variable numbers of radiating actin bundles; if these bundles extend significantly from the lamellipodium, they are named filopodia, while bundles of actin embedded into the lamellipodium are often referred to as microspikes (Figure 1, A and B).

Not all cells are suitable for the study of lamellipodia, owing to their sensitivity to fixation or to a preponderance of ruffling cell edges, which complicate structural investigations. Examples of commonly used lines for the study of lamellipodia include mouse melanoma cells (B16), mouse fibroblasts (NIH3T3), rat fibroblasts (3Y1, Rat2), fish fibroblasts (CAR), macrophage cells (Bac), and neuronal cells (NG108, PC12).

To facilitate structural studies, it is often necessary to induce extensive lamellipodia in cells. There are different ways to stimulate cell protrusion. These include plating the cells onto different substrata such as poly-L-lysine, laminin, or fibronectin (Figure 2); the use of drug treatments such as aluminum fluoride (a stimulator of G-proteins) and phorbol esters; or microinjection or transfection with different proteins. For example, microinjection of low concentrations of Rac1 and local or global application of relaxing drugs like myosin inhibitor ML7 or Rho kinase inhibitor Y27632 lead to hyper motile phenotypes with extensive lamellipodia.

\section{EXPLORATION OF LAMELLIPODIUM FUNCTION AT THE CELLULAR LEVEL: LIGHT MICROSCOPY}

Light microscopy resolves detail in the range of 300-500 nm. In bright-field microscopy, both phase-contrast and Nomarski interference are used for the observation of lamellipodia. Resolution is improved by the application of digital background subtraction strategies. Two basic methods of fluorescence microscopy, observation of fixed cells by classical immunofluorescence and live-cell microscopy using fluorescent probes, are now employed for the observation of lamellipodia (8).

Immunofluorescence on fixed cells allows the localization of lamellipodium proteins at a single point in time for each cell, whereas live-cell microscopy facilitates investigations of the dynamics of these proteins in response to different experimental conditions.

\section{Immunofluorescence of Fixed Cells}

Because of the thickness of the lamellipodium (100-200 $\mathrm{nm})$ and its consequent fragility, specific methods of preparation are required to preserve the structure, organization, and correct localization of actin and resident proteins for microscopic observation. For high-resolution analysis, cells should be plated on glass coverslips. Here considerations of cell density, substrates, and mode of cell stimulation, if any, are important. 
There are mainly three ways to process cells to retain their structural organization: fixation by cross-linking reagents, by precipitation, and by freezing. Chemical fixation is the only practical method for immunofluorescence microscopy because the cells must be stabilized enough to resist distortions during the labeling steps. In one generally useful protocol, extraction and fixation are performed in a two-step procedure (9). (i) A mixture of formaldehyde, glutaraldehyde, and Triton in a specific "Cytoskeleton Buffer" is used for 3 min to pre-fix and extract cells (9). (ii) This is followed by post-fixation for $30 \mathrm{~min}$ in $4 \%$ formaldehyde. Here the filopodia and lamellipodia of numerous cell types are well preserved. (Suitable protocols with an explanation of the properties of different fixatives can be found in Table 1, Immunofluorescence protocols.)

After permeabilization and fixation, cells are processed for fluorescence staining. Depending on the target protein, different approaches such as immunofluorescence (using specific antibodies) or staining with fluorescently labeled toxins can be used. Immunofluorescence was first applied by Lazarides and colleagues (10) to localize actin in non-muscle cells.

Subsequently, it was found that the toxins phalloidin and phallacidin were ideal probes for labeling actin filaments $(11,12)$. These drugs, extracted from the mushroom Amanita phalloides, are able to bind F-actin with a very high affinity after extraction and fixation of cells. It should be noted that phalloidin does not recognize actin that is in a non-polymer state, however. Incidentally, the drug paclitaxel can be used to visualize microtubules in a similar way to phalloidin.

\section{Exploration of Lamellipodium Dynamics}

Recently, substantial progress in characterizing proteins involved in lamellipodium protrusion has come from the use of GFP to tag proteins involved in motility in combination with live-cell microscopy (13). Before the discovery of GFP in 1994 (14), the only way to visualize cytoskeleton dynamics was via the microinjection of purified and fluorescently labeled proteins. Now, GFP from the jellyfish Aequora victoria has become the tool of choice for visualizing various molecular events in the cell.

Transient transfection is a straightforward procedure: one day before an experiment, cells are transfected with DNA encoding GFP-fused protein and then plated on coverslips and mounted in a temperature regulated chamber on an inverted microscope for direct observation. Using this method, the localization of numerous lamellipodial proteins can be observed. Two examples include a movie of GFPActin transfected into $3 Y 1$ cells plated on poly-L-lysine (Movie 2; Table 1) and a movie of the dynamics of GFPVASP transfected into B16 cells and plated onto laminin (Movie 3; Table 1) (15).

The development of derivatives of GFP $(16,17)$ now allows the use of cyan and yellow variants together with red fluorescent protein to follow re-localization of more than one protein in living cells. Using this approach, signals from two sources can be obtained; for example, microtubules were observed to target early focal adhesions, using GFP-tubulin and RFP-Zyxin (Movie 4; Table 1) (18). 


\section{Biolmaging}

An alternative approach for the observation of the in vivo localization of proteins is microinjection of fluorescently labeled proteins. For example, cells transfected with a GFP-EB1 construct (microtubule tip binding protein) were microinjected with recombinant rhodamin-tubulin and TAMRA-vinculin (Movie 5; Table 1).

The additional advantage of live microscopy is the possibility of manipulating cells under the microscope, either chemically or mechanically, to detect changes in molecular dynamics in response to external stimuli. Examples include the local application of tension (19) or of drugs (20).

\section{Alternative Strategies for Probing Structure-Function Relationships}

Confocal (point scanning or spinning disc technology) and wide-field microscopy combined with deconvolution are two methods widely used to acquire stacks of images at different focal planes ( $\mathrm{z}$ location) that after processing will reveal the 3-D distribution of proteins (see Table 1 for various links on introductory concepts in microscopy). Because confocal and deconvolved images give access to very thin focal planes, co-localization of proteins can be easily determined.

Other techniques extending the possibilities of live microscopy are now used to explore lamellipodial function. Speckle microscopy (Table 1, Clare Waterman-Storer Laboratory) is a tool that reveals and quantifies the assembly, dynamics, movement, and turnover of proteins (21). For example, the analysis of actin filament turnover in lamellipodia of living cells was performed using this method (22). The method uses a very low concentration of fluorescent probes microinjected or transfected into cells. Observation is performed using a wide-field or confocal fluorescent microscope coupled to a low-noise cooled charged-coupled device cam-
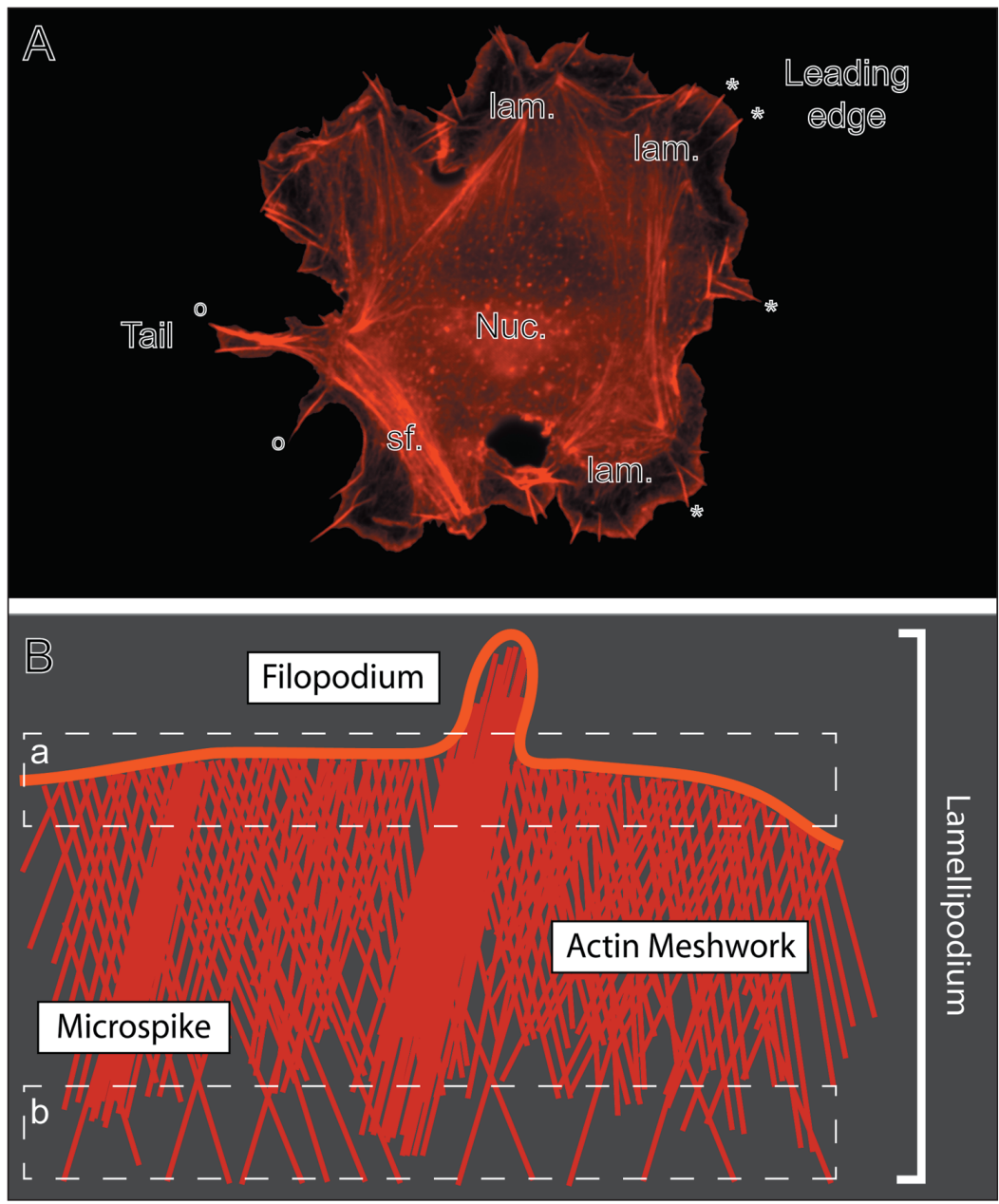

Figure 1. Actin organization in the lamellipodium. (A) Morphology of a spreading cell. Actin staining of B16 melanoma cell plated on laminin and treated with aluminum fluoride. Nuc., nucleus; lam., lamellipodium; sf., stress fibers. Stars show the localization of microspikes, and open circles show the localization of retraction fibers. (B) Schematic representation of subdomains in lamellipodia. (a) Tip of lamellipodium is the major region of actin assembly. (b) Region of major actin disassembly where undegraded actin filaments contribute to the cytoplasmic network.
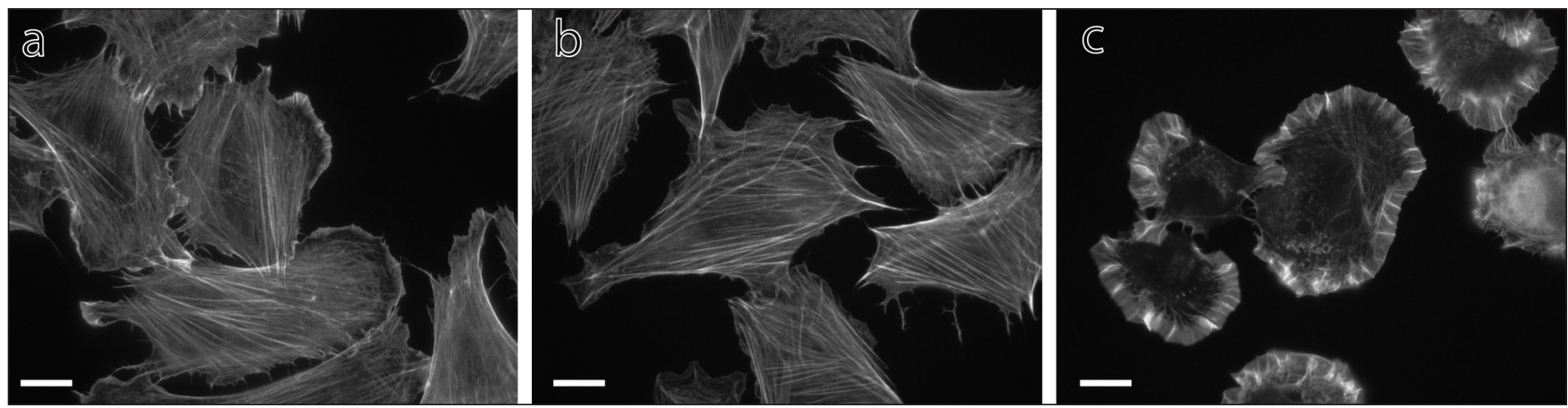

Figure 2. Effects of substratum composition on cell morphology. Actin staining of Rat2 fibroblastic cells plated on different substrata. (a) Laminin-coated coverslips. (b) Fibronectin-coated coverslips. (c) Poly-L-lysine-coated coverslips. Bar $10 \mu \mathrm{m}$. 
Table 1. Resources

Movies:
http://cellix.imolbio.oeaw.ac.at/Publis/BioTech/Movies.htm
Immunofluorescence protocols:
http://www.itg.uiuc.edu/publications/techreports/99-006/
Introduction to light optical microscopy:
http://www.micro.magnet.fsu.edu/primer/index.html

Introductory concepts in fluorescence microscopy: http://www.micro.magnet.fsu.edu/primer/techniques/ fluorescence/fluorhome.html

Introduction to confocal microscopy:

http://www.micro.magnet.fsu.edu/primer/techniques/ confocal/index.html

\section{Clare Waterman-Storer Laboratory: theory of speckle microscopy:} http://www.scripps.edu/cb/waterman/ Flurorescent_speckle_categories.htm

Wolfgang Baumeister Laboratory, Martinsried: http://www.biochem.mpg.de/baumeister/

Gary Borisy Laboratory, Chicago:

http://www.borisylab.nwu.edu/

John Heuser Laboratory, St. Louis:

http://www.heuserlab.wustl.edu/

Free image processing and analysis software, ImageJ: http://rsb.info.nih.gov/ij/

era. The use of very low probe concentrations leads to an incomplete or "speckled" labeling of the structures in the cell (e.g., actin filaments and microtubules) that incorporate the probe. Under these conditions, the movement of the speckles gives information about the turnover of the labeled filaments.

Fluorescent resonance energy transfer (FRET) is also a widely used technique for the detection of protein/protein interactions in vivo, to measure protein interactions discovered by molecular biology and biochemistry. FRET is a process whereby energy from an excited donor fluorophore is transferred to an acceptor fluorophore within a nanometer range. This technique can be used on fixed cells but gives more information when used on living cells (23). For FRET, pairs of fluorophores like GFP/Cyanin 3 or CFP/YFP are used. This approach was used to reveal the small GTPase Rac1 activation dynamics in lamellipodium of living cells (24) using GFP and Alexa-546 probes, or CFP/YFP probes (25).

\section{INCREASING RESOLUTION TO THE MOLECULAR LEVEL: ELECTRON MICROSCOPY}

Light microscopy provides highly informative images, stunning movie sequences, and also visually very appealing pictures of multiple immunofluorescence. However, the details of the organization of filaments within the actin networks, how they are cross-linked in space, and details of where associated proteins are localized in these arrays are beyond the resolution of the light microscope. The improved resolution of electron microscopy is required to address these questions either alone or in combination with light microscopy (26-28).

\section{Classical Approaches}

When it comes to transmission electron microscopy (TEM), most people think of ultra-thin resin-embedded sections. However, this is not necessary for the study of lamellipodia in the electron microscope. Since the lamellipodium is a very thin organelle (100-200 nm), it can be viewed as a whole even in the TEM. All of the techniques presented here use dehydrated whole-cell mounts as introduced by Porter and colleagues (29). To visualize cytoskeletal structures that are covered with membranes and buried in an environment of cytosolic proteins, all the classical approaches rely on a mild detergent extraction. In contrast to the permeabilization protocols employed for light microscopy, these extraction procedures need to remove the membrane and the cytosolic proteins completely to leave only the set of insoluble proteins behind - which is, by definition, the cytoskeleton. Further processing steps then differ considerably according to the chosen method and will be outlined below (8).

Heuser and co-workers (30) and other groups extracted and fixed cytoskeletons and subsequently slammed them onto a copper block at liquid helium temperature for rapid freezing. The frozen samples are transferred to a vacuum evaporator where the ice is allowed to sublime at $-95^{\circ} \mathrm{C}$ ("freeze drying/etching"); after rotary shadowing with metal (to increase the contrast) and carbon, the replicas of these structures are mounted onto grids. A recent application of this method with minor modifications is described previously (31).

The images produced with this technique show a high degree of three-dimensionality (Figure 3a) and good contrast; the three filamentous components of the cytoskeleton can be distinguished solely by their surface morphology. However, when compared to the results obtained using other methods, the linearity of the filaments seems to be lost; highly interconnected filaments dominate. The use of distilled water for washing and of methanol as a cryoprotectant in the original protocols may have contributed to these putative distortions. However, since these treatments were brief, the possibility cannot be excluded that the local growth of ice crystals, which can cause structural damage, during the freezing process or by means of recrystallization at $-95^{\circ} \mathrm{C}$ may have occurred.

Another important advancement in the field of ultrastructural visualization of the cytoskeleton was introduced by Tanya Svitkina from Gary Borisy's laboratory (32): These authors combined an extraction/fixation procedure optimized for cytoskeletons with critical point drying, a technique routinely used for scanning electron microscopy. Together with metal shadowing, this method yielded very impressive im- 


\section{Biolmaging}

ages that were readily accepted by the cytoskeleton community. In brief [a detailed protocol is available from their Web site (Table 1, Gary Borisy Laboratory)], cells grown on coverslips are extracted in the presence of polyethylene glycol to minimize the loss of cytoskeletal material. They are then subjected to a strong fixation followed by dehydration. After, they are transferred to an environment of liquid carbon dioxide and dried by raising the temperature and pressure above the critical values. Here, distortions due to the gas/liquid boundary should be avoided. Platinum replicas of the cytoskeletons, which can be mounted onto grids, are produced in a high vacuum evaporator.

One of the major conclusions (28) reached by this approach was that the actin network of the lamellipodium is extensively branched (Figure $3 \mathrm{~b}$ ); a large number of Y-junctions thought to be the nucleation points of new filaments could be identified. This branched organization is attributed to Arp2/3 (33), a multi-protein complex that could be shown to cause actin filament branching in vitro. However, these results were questioned by the finding that such Y-junctions could be observed even in samples of pure F-actin without
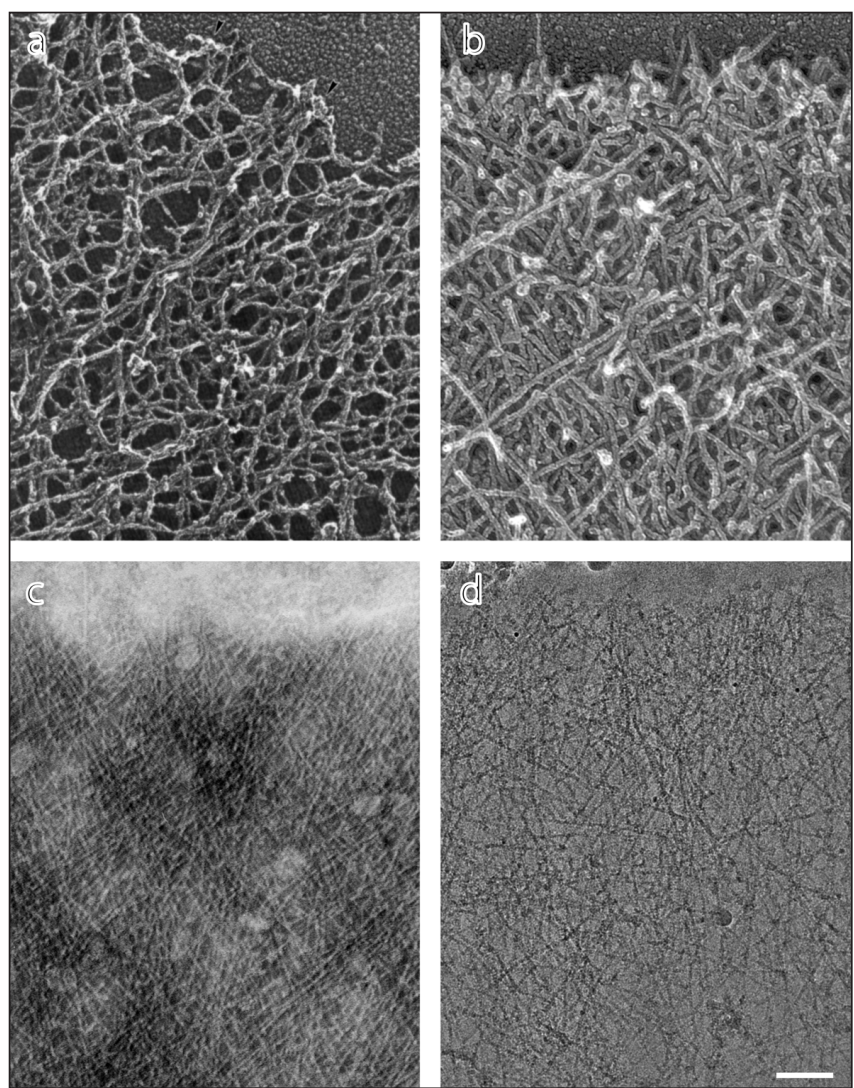

Figure 3. Extracted cytoskeletons from the lamellipodia of keratocytes (a-c) and mouse melanoma cells (d) visualized by TEM after different preparative approaches. (a) Freeze drying and metal shadowing (with permission of John Heuser). (b) Critical point drying and metal shadowing (Reproduced from Reference 28 by copyright permission of Rockefeller University Press). (c) Negative staining (Reproduced from Reference 36 by copyright permission of Rockefeller University Press). (d) Cryo-electron microscopy. Bar $100 \mathrm{~nm}$.
Arp2/3 after the protocol employed by these authors (34). This suggests that the number of branches might be lower than generally expected; a final consensus on this question has not yet been reached.

An alternative approach to visualizing the cytoskeleton networks is provided by the negative staining method. It is very straightforward and requires no special equipment (for a detailed protocol, see Reference 35). Following a brief extraction of the cells in the presence of fixative and a longer post-fixation, the cytoskeletons are rinsed with a solution of a heavy metal salt in a final step. Upon air-drying, these salts form a shell around the filaments, which themselves exclude the electron-dense stain. Air drying (gas/liquid phase boundary) leads to flattening of the sample and is a major concern with this technique. Furthermore, the choice of a stain close to neutral $\mathrm{pH}$ is a critical factor; conventional stains such as uranyl acetate cause more collapse and some distortion and aggregation of the filaments. Nevertheless, this technique has delivered images of lamellipodia with filaments in remarkable order (Figure 3c) $(8,36)$. This suggests that the one-step negative stain method is less damaging than the multiple-step methods described above.

The visualization of cytoskeletal assemblies is not restricted to TEM. A number of unpublished approaches was developed already to visualize these samples by scanning electron microscopy (SEM) and a resolution similar to that achieved by Svitkina's technique (32) could be obtained (R. Wepf, unpublished). However, as for TEM, classical SEM also requires quite extensive processing of the sample so that similar reservations hold about the possibility of introducing artifacts at different steps of the procedure. These might be obviated by the use of cryotechniques, but experience with this is still limited.

\section{Fully Hydrated Cryosamples}

As outlined above, one crucial step for the ultrastructural preservation of actin networks is the processing required for dehydration. In molecular electron microscopy, this problem is now mostly circumvented by observing fully hydrated and unstained specimens at liquid nitrogen or liquid helium temperature. The approach is referred to as "cryoelectron microscopy" (37), and it avoids artifacts introduced by fixation, dehydration, and staining.

To prevent the formation of destructive ice crystals during freezing, samples are cooled at approximately $10^{4} \mathrm{~K} / \mathrm{s}$, which is done in liquid ethane or propane, yielding samples embedded in vitrified or amorphously "frozen" water (38) that have to be maintained below recrystallization temperature in the microscope. Except for some specialized applications (39), there is also no heavy metal stain introduced, so the contrast in the final image relies mainly on the phase contrast by the biological material and is therefore very low; it is defined by the amount of defocus (38). An additional problem is the damage of the hydrated biological matter and the surrounding vitrified water by the electron beam; the radiation that samples are exposed to in conventional TEM was compared to a nuclear explosion. Practically, this means that the electron dose has to be kept below a certain thres- 
hold, which is of particular importance if multiple exposures are to be taken.

The application of this technique to larger structures or even whole cells has so far been limited (40), but two recent publications showed the feasibility of visualizing cytoskeletal assemblies in quick frozen samples. Resch et al. (34) demonstrated that the investigation of fixed and frozen hydrated cytoskeletons can yield high-resolution images of actin assemblies in lamellipodia (Figure 3d), filopodia, and further inside the cell. Nevertheless, this approach is still limited by the extraction, which is necessary to visualize the networks clearly; artifactual modifications of the networks by the loss of actin or its cross-linking proteins cannot be excluded entirely.

A technique presented in a recent publication by Medalia et al. (41) might help to address this issue along with the problem of resolving the 3-D relationship of the structures inside the lamellipodium. In their work on cryo-electron tomography, the authors from the Baumeister laboratory (41) in Munich presented 3-D reconstructions from the periphery of Dictyostelium cells grown on electron microscopy grids, showing dense actin networks and their connections, intracellular membrane systems, ribosomes, and other macromolecular complexes.

The principle behind electron tomography is to take images of the same sample site at many different tilt angles (e.g., from $-60^{\circ}$ to $+60^{\circ}$ at $1.5^{\circ}$ increments) around one or two axes. Backprojection algorithms can convert the aligned stack of images into a 3-D volume. To improve the still relatively low signal (low electron exposure) for visualization, advanced noise reduction algorithms have to be applied. The next obvious steps are to apply this system to higher eukaryotic cells along with light microscopic correlation to obtain data where structure and function can be correlated as well as to establish an in silico test system to confirm the validity of the image processing methods used down to a certain resolution limit.

\section{Localization of Proteins in the Electron Microscope}

The principles behind immunolocalization of proteins by electron microscopy are the same as outlined above for light microscopy. However, markers other than fluorescent dyes have to be used for electron microscopy. For TEM, almost exclusively gold colloids with a particle size of 5-20 nm are used; these electron-dense markers give a very strong and unambiguous signal. Compared to light microscopy, additional difficulties can arise from the stronger fixation protocols employed for electron microscopy, which lead to a loss of antigenicity. A comprehensive introduction on immunocytochemical methods in electron microscopy and the problems one faces there is described previously (42).

Examples on successful immunolabeling of conventionally prepared cytoskeletons can be found in Heuser and Kirschner (30), who identified intermediate filaments by a selective thickening by the antibody binding, Svitkina and Borisy (28) on Arp2/3 with critical point drying technique, and Rottner et al. (15) against VASP in combination with the negative staining approach.
For noninvasive techniques such as cryo-electron tomography, other approaches to localize proteins have to be sought. In this context, Medalia et al. (41) suggest the microinjection of gold-tagged primary antibodies as well as the approach presented in Frangakis et al. (43), which relies on the identification of macromolecular complexes based on their shape in electron tomograms. This second approach is still limited to larger macromolecular assemblies $(>500 \mathrm{kDa})$ than the ones usually found in lamellipodia.

\section{CONCLUSION}

Both light and electron microscopy are indispensable tools for investigating and understanding the mechanisms of lamellipodial protrusion. However, one overriding requirement for all methods should be kept in mind. The preservation of structural features needs to be as close to nature as possible. Unfortunately, this primary goal is often forgotten when the images presented are visually appealing and correspond to what one hopes to see. This is especially true in electron microscopy, and one has to be aware of the advantages and the drawbacks of the individual methods of specimen preparation. Checking fixation procedures and other treatments by comparing cells before and after in the light microscope is a useful precautionary step.

While light microscopy allows a unique insight into the dynamics of crawling cells, by visualizing the distribution of one or more proteins by injected or genetically engineered fluorescent proteins, electron microscopy contributes by improved resolution of structures down to a molecular level. Improvements in digital imaging and in camera sensitivity will give the opportunity to perform confocal (or wide-field deconvolved) time-lapse analysis to reveal the 3-D dynamic of proteins in cells (4-D microscopy). When combined with novel minimally invasive techniques for electron microscopy (such as cyro-electron tomography on whole cells), this will allow us to extend our knowledge of the motor of crawling cells.

\section{ACKNOWLEDGMENTS}

We would like to thank Vic Small for the critical reading of the manuscript, John Heuser, Irina Kaverina, Olga Krylyshkina, Vic Small, and Tatyana Svitkina for the permission to use their images and movies, Roger Wepf and Gareth Griffiths for the helpful discussion, and Johanna Prast and Maria Schmittner for the technical support. E.V. and G.P.R. are supported by project nos. 14660-PAT and P-15710, respectively, of the Austrian Science Foundation.

\section{REFERENCES}

1.Bray, D. 2000. Cell Movements; From Molecules to Motility, 2nd ed. Garland Publishing, New York.

2.Small, J.V. 1995. Getting the actin filaments straight: nucleation-release or treadmilling? Trends Cell Biol. 5:52-55.

3.Small, J.V., K. Rottner, and I. Kaverina. 1999. Functional design in the actin cytoskeleton. Curr. Opin. Cell Biol. 11:54-60. 
4.Ballestrem, C., B. Wehrle-Haller, and B.A. Imhof. 1998. Actin dynamics in living mammalian cells. J. Cell. Sci. 111(Pt 12):1649-1658.

5.Abercrombie, M., J.E. Heaysman, and S.M. Pegrum. 1970. The locomotion of fibroblasts in culture. I. Movements of the leading edge. Exp. Cell Res. 59:393-398.

6.Abercrombie, M., J.E. Heaysman, and S.M. Pegrum. 1971. The locomotion of fibroblasts in culture. IV. Electron microscopy of the leading lamella. Exp. Cell Res. 67:359-367.

7.Small, J.V., T. Stradal, E. Vignal, and K. Rottner. 2002. The lamellipodium: where motility begins. Trends Cell Biol. 12:112-120.

8.Small, J.V., K. Rottner, P. Hahne, and K.I. Anderson. 1999. Visualising the actin Cytoskeleton. Microsc. Res. Tech. 47:3-17.

9.Mies, B., K. Rottner, and J.V. Small. 1998. Multiple immunofluorescence of the cytoskeleton, p. 469-476. In J.E. Celis (Ed.), Cell Biology, A Laboratory Handbook. Academic Press, New York.

10.Lazarides, E. and K. Weber. 1974. Actin antibody: the specific visualization of actin filaments in non-muscle cells. Proc. Natl. Acad. Sci. USA 71:2268-2272.

11.Faulstich, H., S. Zobeley, G. Rinnerthaler, and J.V. Small. 1988. Fluorescent phallotoxins as probes for filamentous actin. J. Muscle Res. Cell Motil. 9:370-383.

12.Small, J.V., S. Zobeley, G. Rinnerthaler, and H. Faulstich. 1988. Coumarin-phalloidin: a new actin probe permitting triple immunofluorescence microscopy of the cytoskeleton. J. Cell Sci. 89(Pt 1):21-24.

13.Ludin, B. and A. Matus. 1998. GFP illuminates the cytoskeleton. Trends Cell Biol. 8:72-77.

14.Chalfie, M., Y. Tu, G. Euskirchen, W.W. Ward, and D.C. Prasher. 1994. Green fluorescent protein as a marker for gene expression. Science 263:802-805.

15.Rottner, K., B. Behrendt, J.V. Small, and J. Wehland. 1999. VASP dynamics during lamellipodia protrusion. Nat. Cell Biol. 1:321-322.

16.Mitra, R.D., C.M. Silva, and D.C. Youvan. 1996. Fluorescence resonance energy transfer between blue-emitting and red-shifted excitation derivatives of the green fluorescent protein. Gene 173:13-17.

17.Rekas, A., J.R. Alattia, T. Nagai, A. Miyawaki, and M. Ikura. 2002. Crystal structure of venus, a yellow fluorescent protein with improved maturation and reduced environmental sensitivity. J. Biol. Chem. 277:5057350578 .

18.Kaverina, I., K. Rottner, and J.V. Small. 1998. Targeting, capture, and stabilization of microtubules at early focal adhesions. J. Cell Biol. 142:181190 .

19.Kaverina, I., O. Krylyshkina, K. Beningo, K. Anderson, Y.L. Wang, and J.V. Small. 2002. Tensile stress stimulates microtubule outgrowth in living cells. J. Cell Sci. 115:2283-2291.

20.Kaverina, I., O. Krylyshkina, M. Gimona, K. Beningo, Y.L. Wang, and J.V. Small. 2000. Enforced polarisation and locomotion of fibroblasts lacking microtubules. Curr. Biol. 10:739-742.

21.Waterman-Storer, C.M., A. Desai, J.C. Bulinski, and E.D. Salmon. 1998. Fluorescent speckle microscopy, a method to visualize the dynamics of protein assemblies in living cells. Curr. Biol. 8:1227-1230.

22.Watanabe, N. and T.J. Mitchison. 2002. Single-molecule speckle analysis of actin filament turnover in lamellipodia. Science 295:1083-1086.

23.Wouters, F.S., P.J. Verveer, and P.I. Bastiaens. 2001. Imaging biochemistry inside cells. Trends Cell Biol. 11:203-211.

24.Kraynov, V.S., C. Chamberlain, G.M. Bokoch, M.A. Schwartz, S. Slabaugh, and K.M. Hahn. 2000. Localized Rac activation dynamics visualized in living cells. Science 290:333-337.

25.Itoh, R.E., K. Kurokawa, Y. Ohba, H. Yoshizaki, N. Mochizuki, and M. Matsuda. 2002. Activation of rac and cdc42 video imaged by fluorescent resonance energy transfer-based single-molecule probes in the membrane of living cells. Mol. Cell Biol. 22:6582-6591.

26.Resch, G.P., K.N. Goldie, A. Krebs, A. Hoenger, and J.V. Small. 2002. Visualisation of the actin cytoskeleton by cryo-electron microscopy. J. Cell Sci. 115:1877-1882.

27.Rinnerthaler, G., M. Herzog, M. Klappacher, H. Kunka, and J.V. Small. 1991. Leading edge movement and ultrastructure in mouse macrophages. J. Struct. Biol. 106:1-16.

28.Svitkina, T.M. and G.G. Borisy. 1999. Arp2/3 complex and actin depolymerizing factor/cofilin in dendritic organization and treadmilling of actin filament array in lamellipodia. J. Cell Biol. 145:1009-1026.

29.Buckley, I.K. and K.R. Porter. 1975. Electron microscopy of critical point dried whole cultured cells. J. Microsc. 104:107-120.
30.Heuser, J.E. and M.W. Kirschner. 1980. Filament organization revealed in platinum replicas of freeze-dried cytoskeletons. J. Cell Biol. 86:212-234

31.Flanagan, L.A., J. Chou, H. Falet, R. Neujahr, J.H. Hartwig, and T.P. Stossel. 2001. Filamin A, the Arp2/3 complex, and the morphology and function of cortical actin filaments in human melanoma cells. J. Cell Biol. 155:511-517.

32.Svitkina, T.M., A.B. Verkhovsky, and G.G. Borisy. 1995. Improved procedures for electron microscopic visualization of the cytoskeleton of cultured cells. J. Struct. Biol. 115:290-303.

33.Pollard, T.D., L. Blanchoin, and R.D. Mullins. 2000. Molecular mechanisms controlling actin filament dynamics in nonmuscle cells. Annu. Rev. Biophys. Biomol. Struct. 29:545-576.

34.Resch, G.P., K.N. Goldie, A. Hoenger, and J.V. Small. 2002. Pure F-actin networks are distorted and branched by steps in the critical-point drying method. J. Struct. Biol. 137:305-312.

35.Small, J.V. and A. Sechi. 1998. Whole-mount electron microscopy of the cytoskeleton: negative staining methods, p. 285-291. In J.E. Celis (Ed.), Cell Biology, A Laboratory Handbook. Academic Press, New York.

36.Small, J.V., M. Herzog, and K. Anderson. 1995. Actin filament organization in the fish keratocyte lamellipodium. J. Cell Biol. 129:1275-1286.

37.Dubochet, J., M. Adrian, J.J. Chang, J.C. Homo, J. Lepault, A.W. McDowall, and P. Schultz. 1988. Cryo-electron microscopy of vitrified specimens. Q. Rev. Biophys. 21:129-228.

38.Roos, N. and A.J. Morgan. 1990. Cryopreparation of Thin Biological Specimen for Electron Microscopy: Methods and Applications. Oxford University Press, New York.

39.Adrian, M., J. Dubochet, S.D. Fuller, and J.R. Harris. 1998. Cryo-negative staining. Micron 29:145-160.

40.McIntosh, J.R. 2001. Electron microscopy of cells: a new beginning for a new century. J. Cell Biol. 153:F25-F32.

41.Medalia, O., I. Weber, A.S. Frangakis, D. Nicastro, G. Gerisch, and W. Baumeister. 2002. Macromolecular architecture in eukaryotic cells visualized by cryoelectron tomography. Science 298:1209-1213.

42.Griffiths, G. 1993. Fine Structure Immunocytochemistry. Springer-Verlag, Berlin.

43.Frangakis, A.S., J. Bohm, F. Forster, S. Nickell, D. Nicastro, D. Typke, R. Hegerl, and W. Baumeister. 2002. Identification of macromolecular complexes in cryoelectron tomograms of phantom cells. Proc. Natl. Acad. Sci. USA 99:14153-14158.

Address correspondence to Dr. Emmanuel Vignal, Austrian Academy of Sciences, Institute of Molecular Biology, Department of Cell Biology, Billrothstrasse, 11, A-5020 Salzburg, Austria. e-mail: evignal@imb.oeaw.ac.at

Suggestions for contributions to the Biolmaging feature are welcomed by its editor, Dr. Steve Paddock (paddock@facstaff.wisc.edu) 